10-15-1990

\title{
Heterotic Conformal Field Theory and Gepner's Construction
}

\author{
Darwin Chang \\ University of Maryland - College Park
}

Asim Gangopadhyaya

Loyola University Chicago, agangop@luc.edu

Alok Kumar

Laboratoire de Physique Theorique

Jin Wang

University of Illinois at Urbana-Champaign

Follow this and additional works at: https://ecommons.luc.edu/physics_facpubs

Part of the Other Physics Commons, and the Quantum Physics Commons

\section{Recommended Citation}

Chang, D., Gangopadhyaya, A., Kumar, A., and Wang, J. (1990). Heterotic conformal field theory and Gepner's construction. Physical Review D, 42(8), 2694-2703.

This Article is brought to you for free and open access by the Faculty Publications and Other Works by Department at Loyola eCommons. It has been accepted for inclusion in Physics: Faculty Publications and Other Works by an authorized administrator of Loyola eCommons. For more information, please contact ecommons@luc.edu. \section{(c) (i) $\Theta$}

This work is licensed under a Creative Commons Attribution-Noncommercial-No Derivative Works 3.0 License. (c) American Physical Society, 1990. 


\title{
Heterotic conformal field theory and Gepner's construction
}

\author{
Darwin Chang \\ Department of Physics and Astronomy, Northwestern University, Evanston, Illinois 60208 \\ Asim Gangopadhyaya \\ Department of Physics, Loyola University, Chicago, Illinois 60626 \\ Alok Kumar \\ Laboratoire de Physique Théorique, 24 Rue Lhomond, 75231 Paris, CEDEX 05, France
}

Jin Wang

Department of Physics, University of Illinois at Urbana-Champaign, Urbana-Champaign, Illinois 61801

(Received 23 February 1990)

\begin{abstract}
We discuss some general properties of heterotic conformal field theory in which conformal anomalies $c$ are different for the left-moving and right-moving sectors. It is precisely this type of theory that can be applied immediately to the construction of heterotic string theory. We discuss a general way of constructing such a theory using free fermions. The construction is then applied to generalize Gepner's construction of superstring solutions using the tensor products of $N=2$ superconformal field theories.
\end{abstract}

\section{INTRODUCTION}

Since consistent superstring solutions were discovered in ten dimensions ${ }^{1}$ in 1984 which triggered the theoretical fervor in string theory in recent years, there has been a lot of progress in constructing consistent superstring solutions in $D<10$ dimensions. Most notably, there have been the Calabi-Yau compactification approach, ${ }^{2}$ the orbifold compactification approach, ${ }^{3}$ the covariant lattice approach, ${ }^{4}$ fermionic approach, ${ }^{5}$ and others. ${ }^{6}$ All these constructions may be related to each other through boson-fermion equivalence or a more complicated equivalence which is not yet well understood. Many of these relations are, of course, already pointed out in the literature.

Underlying every string solution is, of course, a conformal or superconformal theory to guarantee the consistency of the huge gauge symmetry in string theory. Therefore, one way of classifying the string solutions is to uncover the structure of all possible conformal field theories (CFT's). As a stepping stone, one would like to classify the simpler rational conformal field theory (RCFT) in which the partition function can be written as a finite sum of products of holomorphic (left-moving) parts and antiholomorphic (right-moving) parts. It is conjectured ${ }^{7}$ that these holomorphic parts actually correspond to some characters of an extended conformal algebra $A_{L}$ and similarly for the right-handed sector with an extended algebra $A_{R}$. There has been great progress in this direction. ${ }^{8}$ Most of this progress applies readily to theories in which the conformal anomaly in the left-handed sector $c_{L}$ is the same as that in the right-handed sector $c_{R}$. We shall call these type-I CFT's. It is interesting to ask to what extent these results can be applied to cases in which
$c_{L} \neq c_{R}$. In particular, in phenomenologically more interesting heterotic string theory, ${ }^{9}$ a superconformal field theory is used in the left-handed sector while only a conformal field is employed in the right-handed sector. In order to guarantee to consistency of the world-sheet gauge symmetry, one needs the underlying conformal field theories (excluding the ghost contributions and taking the light-cone gauge) to have $c_{L}=12$ and $c_{R}=24$. Therefore any understanding of conformal field theory with $c_{L} \neq c_{R}$ will be useful in the construction of more heterotic string theories. We shall call them heterotic conformal field theories (HCFT's).

Most of the results about the classification of CFT can be applied to HCFT with some modification. However, there are some distinct features that mark their difference. In Sec. II of this paper we emphasize some of these differences and point out some interesting unsolved problems. Clearly, it is much harder to construct HCFT because it is much more difficult to satisfy the condition of modular invariance. This is unlike the type-II case where one can always have a diagonal invariant. However, recent developments taught us that the condition of modular invariance can be easily solved if one restricts oneself to the free fermionic construction. ${ }^{5}$ In Sec. III we apply the free fermionic construction to generate a whole class of HCFT's. Some comments on the deformation of these theories using Thirring interactions are also in order. It is clear that one can do the same thing using the bosonic approach as well. To demonstrate the use of these constructions, we apply them to Gepner's construction of superstring solutions. Gepner's construction involves two steps. One first constructs a type-II superstring model in $d<10$ dimensions with internal sectors added on to saturate the conformal anomaly. The inter- 
nal sectors that Gepner uses are tensor products of minimal $N=2$ superconformal field theories. It has been pointed out ${ }^{10}$ that in order to obtain space-time supersymmetry it is necessary that the spectrum form representations of $N=2$ superconformal algebra. Gepner has devised an elaborate scheme to satisfy modular invariance and spin-statistics relations (called the $\beta$ method). Then the second step is to convert the resulting type-II theory by a "heterotic replacement" in which the partition function of the right-handed fermions with spacetime index are replaced by the characters of an extended algebra which has the same modular transformation property. Gepner found two ways of doing this. It is clear that our modular-invariant HCFT can be readily applied to the second step and generates more general solutions. In Sec. III we review Gepner's construction and define some notations for future need. In Sec. IV, we apply the HCFT that we generated in Sec. III to the construction. Some examples are given in detail for illustrative purposes. Some technical details about Gepner's construction that do not exist in the literature are included in Appendix $\mathrm{C}$ for reference.

\section{HETEROTIC CONFORMAL FIELD THEORY}

A rational conformal field theory is defined by a partition function which is a finite sum such as

$$
Z_{R}=\sum_{i, j} \chi_{i} \bar{\chi}_{j}
$$

where $\chi$ is a holomorphic function and $\bar{\chi}$ is an antiholomorphic function. $\chi$ 's $\left(\bar{\chi}^{\prime}\right.$ s) are characters of an extended conformal algebra $A_{L}\left(A_{R}\right)$ for the left- (right-)handed sector. If one assumes (as we are going to do from now on) that one of the characters is the character $\chi_{0}$, generated from the vacuum state for the $A_{L}$ algebra, then the conformal anomaly of the holomorphic sector can be determined as follows. If one expands each of the characters in $Z_{R}$ as a Laurent series in $q=\exp (2 \pi i \tau)$, then the one with the smallest leading exponent corresponds to the vacuum character and its leading exponent is $c_{L} / 24$. Similarly, one can determine the vacuum character $\bar{\chi}_{0}$ and conformal anomaly $c_{R}$ of the antiholomorphic sector. If $c_{L}=c_{R}, A_{L}=A_{R}$, and $Z_{R}$ contains the term $\chi_{0} \bar{\chi}_{0}$, then it corresponds to the usual rational conformal field theory defined in the literature. ${ }^{8}$ We shall call this type-I RCFT. There are diagonal and nondiagonal partition functions in this type. The nondiagonal ones are necessarily more difficult to find because the modular-invariant conditions are more difficult to satisfy. Here we are more interested in the case when $c_{L}$ is not equal to $c_{R}$. We shall call these the heterotic type.

One of the immediate consequences of the heteroticity is that the partition function cannot contain the term $\chi_{0} \bar{\chi}_{0}$. That means that the usual assumption about the uniqueness of the vacuum state $(0,0)$ does not apply. The modular invariance is even more difficult to satisfy than the nondiagonal type-I RCFT. First, the partition function contains the terms $\chi_{0} \bar{\chi}_{\bar{h}}+\chi_{h} \bar{\chi}_{0}$, where $h$ is defined by the leading exponent $\left(h-c_{L} / 24\right)$ of the corresponding character, as is $\bar{h}$. In order to satisfy modular invariance under $\tau \rightarrow \tau+1$, one needs

$$
\begin{aligned}
& c_{L} / 24-h=c_{R} / 24+\text { integer }, \\
& c_{R} / 24-\bar{h}=c_{L} / 24+\text { integer } .
\end{aligned}
$$

These conditions are very difficult to arrange. For a given $A_{L}$, the corresponding $c_{L}$ and a finite list of $h$ 's are fixed. Then Eq. (2) gives a very strong restriction on allowed $c_{R}$. One can look for the $A_{R}$ 's which have the proper $c_{R}$. Then in order to have a modular-invariant partition function, one needs to make sure that $A_{\underline{R}}$ contains a primary field with conformal weight $\bar{h}$ that satisfies Eq. (3).

However, from experience in the fermionic or bosonic constructions of heterotic (super)string solution in less than 10 dimensions, one knows that the modularinvariance conditions for these cases can be solved explicitly. Many schemes for generating (super)string solutions have been worked out. ${ }^{2-5}$ Therefore, all these techniques can be easily converted into schemes for generating HCFT. In the next section, we describe the free fermionic techniques for this purpose.

\section{FREE FERMIONIC CONSTRUCTION OF HCFT}

In this section we use free fermionic construction to build heterotic conformal field theories. We shall adopt the notation of Antoniadis, Bachas, and Kounnas ${ }^{5}$ (ABK) for simplicity. A short review of this construction is included in Appendix A. Suppose we build a conformal field theory with conformal anomalies $c_{L}$ and $c_{R}$. In the free fermionic construction we use a free fermion to saturate these anomalies. Therefore, we need $n_{L}=2 c_{L}$ lefthanded fermions $\psi_{L}^{i}$ and $n_{R}=2 c_{R}$ right-handed fermions $\chi_{R}^{i}$. For a real fermion, which is what we shall confine our discussions to, the boundary conditions can be either periodic $(P)$ or antiperiodic $(A)$. Given $n_{L}$ and $n_{R}$ fermions, a boundary condition for the set is defined by specifying the boundary conditions for each of these fermions. It can be represented by a subset which contains all the fermions that have periodic boundary conditions. A modular-invariant solution can be defined by a consistent collection $\Xi$ of such subsets. This collection can in turn be generated by a set of basis elements. For heterotic string theory, these consistency conditions have been worked out by many authors. ${ }^{5}$ Since heterotic string theory is just an example of HCFT, these conditions are not necessarily applicable to our case. However, it turns out the conditions for modular invariance are almost identical to those derived by ABK. These conditions are displayed in Appendix A. Here we shall be content with simply describing the set of solutions we have for later application. A typical example has been worked out in detail in Appendix A for illustration.

To provide examples of this construction and ultimately apply it to Gepner's construction, we list, in Table I, the models we find for $\Delta c=12$ and $n_{L}=2,4,6$. This list will be useful for Gepner's construction in $D=4,6$, or 8 space-time dimensions, respectively. We cannot prove that we have exhausted all possible models using a free fermion in each case. However, we believe that our list is 
TABLE I. HCFT's generated by free fermionic construction.

\begin{tabular}{lcccc}
\hline \hline Dimension & Model & Tachyon & $\boldsymbol{A}_{R}$ & $\begin{array}{c}\text { Gauge } \\
\text { Boson }\end{array}$ \\
\hline$D=4$ & $\boldsymbol{M}_{1}^{4}$ & 26 & $\mathrm{SO}(26)$ & 325 \\
& $\boldsymbol{M}_{2}^{4}$ & 10 & $\mathrm{E}_{8} \times \mathrm{SO}(10)$ & 293 \\
& $\boldsymbol{M}_{3}^{4}$ & 2 & $\mathrm{SO}(2) \times \mathrm{SO}(24)$ & 277 \\
$D=6$ & & & & \\
& $\boldsymbol{M}_{1}^{6}$ & 28 & $\mathrm{SO}(28)$ & 378 \\
& $\boldsymbol{M}_{2}^{6}$ & 12 & $\mathrm{E}_{8} \times \mathrm{SO}(12)$ & 314 \\
& $\boldsymbol{M}_{3}^{6}$ & 4 & $\mathrm{SO}(4) \times \mathrm{SO}(24)$ & 282 \\
& $\boldsymbol{M}_{4}^{6}$ & 0 & $\mathrm{E}_{7} \times \mathrm{E}_{7}$ & 266 \\
$D=8$ & & & & \\
& $\boldsymbol{M}_{1}^{8}$ & 30 & $\mathrm{SO}(30)$ & 435 \\
& $\boldsymbol{M}_{2}^{8}$ & 14 & $\mathrm{E}_{8} \times \mathrm{SO}(14)$ & 339 \\
& $\boldsymbol{M}_{3}^{8}$ & 6 & $\mathrm{SO}(6) \times \mathrm{SO}(24)$ & 291 \\
& $\boldsymbol{M}_{4}^{8}$ & 2 & $\mathrm{E}_{7} \times \mathrm{E}_{7} \times \mathrm{U}(1)$ & 267 \\
& $\boldsymbol{M}_{5}^{8}$ & 0 & $\mathrm{SU}(16)$ & 255 \\
\hline \hline
\end{tabular}

complete in this context, as we made an extensive search for other models without success.

The partition functions of these models can be written in a very compact form. The antiholomorphic (lefthanded) part can be expressed in terms of $\mathrm{SO}(d)$ character at level $k=1$. For even $d$, there are four integrable highest-weight representations which are the singlet $(0)$, vector $(V)$, spinor $(s)$, and antispinor $(\bar{s})$. Their characters can be written in terms of $\theta$ functions. Define $a=\left(\theta_{2} / \eta\right), b=\left(\theta_{3} / \eta\right)$, and $c=\left(\theta_{4} / \eta\right)$. Two interesting identities are $a b c=2$ and $a^{4}=b^{4}-c^{4}$. The $\mathrm{SO}(d)$ characters with $d=2 n$ can be written as

$$
\begin{aligned}
& \chi_{0}^{2 n}=\frac{1}{2}\left(b^{n}+c^{n}\right), \\
& \chi_{V}^{2 n}=\frac{1}{2}\left(b^{n}-c^{n}\right), \\
& \chi_{s}^{2 n}=\chi_{\bar{s}}^{2 n}=\frac{1}{2} a^{n} .
\end{aligned}
$$

They can also be written as the sums of products of level-2 classical $\theta$ functions which will be useful for $N=2$ superconformal model construction later. They are, of course, also related to the usual level-1 classical $\theta$ function of $\mathrm{SO}(2 n)$ Lie algebra $\theta_{\lambda}$ through the usual relation $\chi_{\lambda}^{d}=\theta_{\lambda}(\tau) /[\eta(\tau)]^{n}$.

Denote the set of fermions $F$ as $\left(\psi_{L}^{1} \cdots \psi_{L}^{d} \mid \chi_{R}^{1} \cdots \chi_{R}^{d+24}\right)$, where the dimension of space-time is $D=d+2$. In $D=4$ dimensions, we could find only three solutions. First, if one used only one basis $F$, then one would have the model with a right-handed chiral algebra $A_{R}=\mathrm{SO}(26)$. We will call this model $M_{1}^{4}$. If one adds a second basis $b_{1}=\left(\chi_{R}^{1} \cdots \chi_{R}^{16}\right)$ then one gets the $M_{2}^{4}$ model with $A_{R}=\mathrm{E}_{8} \times \mathrm{SO}(10)$. If another basis $b_{2}=\left(\chi_{R}^{1} \cdots \chi_{R}^{8}, \chi_{R}^{17} \cdots \chi_{R}^{24}\right)$ is added to the set, one finds the $M_{3}^{4}$ model with $A_{R}=\mathrm{SO}(2) \times \mathrm{SO}(24)$.

In $D=6$ dimension, there are four solutions. Take $F$, $b_{1}$, and $b_{2}$ to be the same as in the $D=4$ case, and add $b_{3}=\left(\chi_{R}^{1} \cdots \chi_{R}^{4}, \chi_{R}^{9} \cdots \chi_{R}^{12}, \chi_{R}^{17} \cdots \chi_{R}^{20}, \chi_{R}^{25} \cdots \chi_{R}^{28}\right)$ to the list of bases. The model with just one basis $F$ gives $A_{R}=\mathrm{SO}(28)$; with $F$ and $b_{1}$, we get $A_{R}=\mathrm{E}_{8} \times \mathrm{SO}(12)$; with $F, b_{1}$, and $b_{2}, A_{R}=\mathrm{SO}(4) \times \mathrm{SO}(24)$; and with $F, b_{1}$, $b_{2}$, and $b_{3}$, one ends up with $A_{R}=\mathrm{E}_{7} \times \mathrm{E}_{7}$. We denote them collectively by $M_{i}^{6}, i=1,2,3,4$.

For $D=8$, there are five solutions. Take $F, b_{1}, b_{2}, b_{3}$ to be the same as the $D=6$ case and $b_{4}=\left(\chi_{R}^{i} \mid i=4 n+1\right.$ or $4 n+2 ; i \leq 6) . M_{1}^{8}$ has only one basis $F, A_{R}=\mathrm{SO}(30) . M_{2}^{8}$ uses $F$ and $b_{1}$ as bases and $A_{R}=\mathrm{E}_{8} \times \mathrm{SO}(14) . M_{3}^{8}$ uses $F$, $b_{1}, b_{2}$, and $A_{R}=\mathrm{SO}(6) \times \mathrm{SO}(24) . M_{4}^{8}$ uses $F, b_{1}, b_{2}, b_{3}$, and $A_{R}=\mathrm{E}_{7} \times \mathrm{E}_{7} \times \mathrm{U}(1) . M_{5}^{8}$ uses $F, b_{1}, b_{2}, b_{3}, b_{4}$ and one gets $A_{R}=\mathrm{SU}(16)$. These models are all listed in Table I together with the numbers of tachyons and the gauge bosons in $A_{R}$. There are, of course, many other ways of choosing different bases; however, we find that they always reproduce one of these models. We conjecture that this list is complete as far as real fermionic construction is concerned. The case of $M_{3}^{8}$ is worked out in detail in Appendix A as an example.

The partition function for $M_{N}^{d=2 n}$ models can be written as

$$
Z(d, N)=\bar{\chi}_{0}^{d} \chi_{0}^{e}+\bar{\chi}_{V}^{d} \chi_{V}^{e}+\bar{\chi}_{s}^{d} \chi_{s}^{e}+\bar{\chi}_{s}^{d} \chi_{\bar{s}}^{e},
$$

where the "effective" holomorphic characters are

$$
\begin{aligned}
\chi_{0}^{e}=\frac{1}{2^{N}}\{ & b^{12+n}-c^{12+n} \\
& \left.+\left(2^{N-1}-1\right)\left[\left(c^{8}+a^{8}\right) b^{4+n}-\left(b^{8}+a^{8}\right) c^{4+n}\right]\right\},
\end{aligned}
$$

$$
\begin{aligned}
\chi_{V}^{e}=\frac{1}{2^{N}} & \left\{b^{12+n}+c^{12+n}\right. \\
& \left.\quad+\left(2^{N-1}-1\right)\left[\left(c^{8}+a^{8}\right) b^{4+n}+\left(b^{8}+a^{8}\right) c^{4+n}\right]\right\},
\end{aligned}
$$

$\chi_{s}^{e}=\chi_{\bar{s}}^{e}=\frac{1}{2^{N}}\left\{-a^{12+n}-\left(2^{N-1}-1\right)\left[\left(b^{8}+c^{8}\right) a^{4+n}\right]\right\}$

and $1 \leq N \leq n+2$. In fact these partition functions also apply to $d=8$, that is, $(D=10)$-dimensional models. It reproduces all the nonsupersymmetric models listed in Ref. 5, except the tachyon free $\mathrm{SO}(16) \times \mathrm{SO}(16)$ model which corresponds to formally taking the $N \rightarrow \infty$ limit of $Z(d, N)$. Clearly for different $d$ and $N$ the effective holomorphic characters can be identified as the characters of the corresponding $A_{R}$ Kac-Moody algebra. For example, for the $M_{3}^{4}$ model

$$
\begin{aligned}
& \chi_{0}^{e}=\chi_{V}^{\mathrm{SO}(2)} \chi_{0}^{\mathrm{SO}(24)}+\chi_{0}^{\mathrm{SO}(2)} \chi_{s}^{\mathrm{SO}(24)}, \\
& \chi_{V}^{e}=\chi_{0}^{\mathrm{SO}(2)} \chi_{0}^{\mathrm{SO}(24)}+\chi_{V}^{\mathrm{SO}(2)} \chi_{s}^{\mathrm{SO}(24)}, \\
& \chi_{s}^{e}=\chi_{s}^{e}=-\chi_{s}^{\mathrm{SO}(2)} \chi_{s}^{\mathrm{SO}(24)}-\chi_{s}^{\mathrm{SO}(2)} \chi_{V}^{\mathrm{SO}(24)} .
\end{aligned}
$$

Modular invariance can also be checked in this form with the aid of the Jacobi identity. Now that we have constructed a list of HCFT's with $c_{R}-c_{L}=12$, an immediate application of this result is to use these partition functions to facilitate a more general "heterotic replacement" procedure in Gepner's construction of string or superstring solution. Note that Eqs. (8)-(10) in our free fermionic construction will guarantee that $\chi_{0}^{e}$ and $\chi_{V}^{e}$ always 
contribute to $Z(d, N)$ with a positive multiplicity and $\chi_{s}^{e}$, $\chi_{\bar{s}}^{e}$ always contribute with a negative multiplicity. It is actually a consequence of the modular invariance. This property will automatically preserve the spin-statistics relation when we apply this to the heterotic replacement procedure of Gepner's construction.

\section{GEPNER'S CONSTRUCTION}

Gepner ${ }^{6}$ has initiated the construction of string theory in $D \leq 10$ dimensions by using a tensor product of $r$ copies of level $k_{i}, N=2$ minimal superconformal theories as the internal part of the theory that helps saturate the conformal anomaly. The resulting theory can mimic a string theory compactified on a complicated Calabi-Yau manifold. Gepner's construction starts with a type-II theory in $D$ dimensions. To saturate conformal anomaly, one must satisfy

$$
\sum_{j=1}^{r} \frac{3 k_{i}}{k_{i}+2}=\frac{3}{2}(10-D)
$$

This composite $N=2$ model will be represented by $\prod_{i=1}^{r}\left(k_{i}\right)$. An $N=2$ minimum model labeled by $k$ can have four different sectors: one Neveu-Schwarz (NS) sector, two Ramond sectors $P^{ \pm}$, and one twisted sector. The twisted sector does not mix with other sectors under modular transformation and so far does not seem to be useful in model building. There are $(k+1)(k+2) / 2$ primary fields for each of the NS, $P^{+}, P^{-}$sectors. Each of them is labeled by two quantum numbers $(h, Q)$, where $h$ is the conformal weight and $Q$ is the charge. The spectrum is

$$
\left(h_{l, q}^{\lambda}, Q_{l, q}^{\lambda}\right)=\left(\frac{l(l+2)-(q-2 \lambda)^{2}}{4(k+2)}+\frac{1}{2} \lambda^{2}, \frac{q+\lambda k}{k+2}\right),
$$

where $0 \leq l \leq k,-l \leq q \leq l$, and $l+q=0(\bmod 2), \lambda=0$ for the NS sector and $\lambda= \pm \frac{1}{2}$ for the Ramond sector $\left(P^{ \pm}\right)$.

The character corresponding to these $N=2$ primary fields can be defined as ${ }^{11}$

$$
\operatorname{Ch}(\tau, z)=\operatorname{Tr}\left(q^{L_{0}-c / 24} y^{Q}\right)
$$

where $q=e^{2 \pi i \tau}$ and $y=e^{2 \pi i z}$. They are given explicitly by

$$
\begin{aligned}
\mathrm{Ch}_{l, q}^{(k, \lambda)}(\tau, z) & =\sum_{q^{\prime}=-k+1}^{k} C_{l q^{\prime}}^{(k)}(\tau) \\
& \times \Theta_{q^{\prime}(k+2)-q k+2 \lambda k, k(k+2)}\left(\frac{\tau}{2}, \frac{z}{k+2}\right),
\end{aligned}
$$

where the theta functions $\Theta_{m, k}$ are defined by

$$
\Theta_{m, k}(\tau, z)=\sum_{n \in Z+m / 2 k} q^{k n^{2}} y^{k n}
$$

and $C_{l q^{\prime}}^{(k)}(\tau)$ are the string functions of the affine $\mathrm{SU}(2)$ algebra. ${ }^{12}$ They have the symmetry

$$
C_{l, q}^{(k)}(\tau)=C_{l, q+2 k Z}^{(k)}(\tau)=C_{l,-q}^{(k)}(\tau)=C_{k-l, k-q}^{(k)}(\tau)
$$

and $C_{l, q}^{(k)}(\tau)=0$ if $(l-q) \neq 0(\bmod 2)$. Theta functions have a symmetry $\Theta_{m, k}(\tau, z)=\Theta_{m+2 k z, k}(\tau, z)$, which leads to the following relationship among characters:

$$
\begin{aligned}
\mathrm{Ch}_{l, q}^{(k, \lambda)}(\tau, z) & =\mathrm{Ch}_{l,-q}^{(k,-\lambda)}(\tau,-z) \\
& =\mathrm{Ch}_{k-l, k+2+q}^{(k, \lambda)}(\tau, z) \\
& =\mathrm{Ch}_{l, q+2(k+2) Z}^{(k, \lambda)}(\tau, z) .
\end{aligned}
$$

Note that the descendent states in an $N=2$ representation are created by the generators $G^{ \pm}$of the two supersymmetries and a $\mathrm{U}(1)$ current $J$ of the $N=2$ algebra. For the NS sector, the mode expansion of $G^{ \pm}$carries half-integer indices. Under the modular transformation $T: \tau \rightarrow \tau+1, \mathrm{Ch}_{l q}^{(k, 0)}$ will not transform into itself. Therefore it is convenient also to introduce another character $\mathrm{Ch}_{l, q}^{(k, \tilde{0})}$ which is defined by

$$
\mathrm{Ch}_{l, q}^{(k, \tilde{0})}(\tau, z)=\mathrm{Ch}_{l, q}^{(k, 0)}(\tau+1, z)
$$

It is useful to work with the eigenstates of $T: \tau \rightarrow \tau+1$ transformation, which are

$$
\begin{aligned}
\chi_{l, q}^{k,(s=0)}(\tau, z)=\frac{1}{2}[ & \operatorname{Ch}_{l, q}^{(k, 0)}\left(\tau, \frac{z}{k+2}\right] \\
& \left.+e^{-2 \pi i\left(h_{l, q}^{(0)}-c / 24\right)} \operatorname{Ch}_{l, q}^{(k, \tilde{0})}\left(\tau, \frac{z}{k+2}\right)\right], \\
\chi_{l, q}^{k,(s=2)}(\tau, z)=\frac{1}{2}[ & \operatorname{Ch}_{l, q}^{(k, 0)}\left(\tau, \frac{z}{k+2}\right) \\
& \left.-e^{-2 \pi i\left(h_{l, q}^{(0)}-c / 24\right)} \mathrm{Ch}_{l, q}^{(k, \tilde{0})}\left(\tau, \frac{z}{k+2}\right)\right] .
\end{aligned}
$$

These are exactly the same characters as those Gepner uses to obtain modular-invariant partition functions. In general, Gepner's characters are given by ${ }^{6}$

$$
\begin{aligned}
\chi_{l, q}^{k(s)}(\tau, z) & =\sum_{j \bmod k} c_{l, m}^{k}(\tau) \\
& \times \Theta_{2 m+(4 j-s)(k+2), 2 k(k+2)}\left(\tau, \frac{z}{(k+2)}\right),
\end{aligned}
$$

where the index $s$ is defined as $\bmod 4$ and is even in the NS sector and odd in the Ramond ( $R$ ) sector. In $\mathbf{R}$ sectors we found the identities

$$
\mathrm{Ch}_{l, q=1}^{k(=1 / 2)}=\chi_{l, q}^{k(1)}(\tau, z)+\chi_{l, q}^{k(3)}(\tau, z) \text {. }
$$

Under $T$ transformation, now we have the simple form

$$
\chi_{l, q}^{k(s)}(\tau+1,0)=e^{2 \pi i h_{l, q}^{(\lambda)}} \chi_{l, q}^{k(s)}(\tau, 0) .
$$

The $\chi$ characters have the symmetry $\chi_{l, q}^{k(s)}=\chi_{k-l, q+k+2}^{k(s+2)}$, which follows from the relation $\exp \left(2 \pi i h_{l, q}^{(0)}\right)$ $=-\exp \left(2 \pi i h_{k-l, q+k+2}^{(0)}\right)$.

Gepner made the crucial observation that the modular-transformation property of $\chi_{l, q}^{k(s)}$ is the same as the product of an $A_{1}^{(1)}$ character, a level- $(k+2) \theta$ function system and a level-2 $\theta$-function system. The 
$A_{1}^{(1)}$ character, the level- $(k+2)$ and level-2 $\theta$ functions are labeled by $l, q$, and $s$, respectively. Since each of the three pieces transform independently under $S$, the modular-invariant composite characters can be constructed out of the product of the three independent modular invariants $Z=Z_{A} Z_{k+2} Z_{2}$. Gepner's observation means that each modular invariant we constructed for the composite theory gives an immediate way of constructing an $N=2$ modular invariant by using the same numerical linear combination as in $Z_{A} Z_{k+2} Z_{2}$.

To form a type-II string theory we have to take a cross product of $r, N=2$, theories as the internal part and add the space-time part. In $D=d+2$ dimensions, the contribution of the space-time part to the partition function is in the form of the $\mathrm{SO}(d)_{L} \times \mathrm{SO}(d)_{R}$ character. It is useful to know that a level-1 $\mathrm{SO}(d)$ character can be decomposed into the finite sum of products of level-2 $\theta$ functions. For example, for $d=2 N, k=1$, the spinorcharacter can be written as

$$
\Theta_{\mathbf{s}}\left(\tau, z_{i}, 0\right)=\sum_{\substack{n_{i}= \pm 1, \prod n_{i}=1}} \prod_{i} \Theta_{n_{i}, 2}\left(\tau, z_{i}, 0\right)
$$

and $\Pi n_{i}=-1$ for the antispinor character. For the sca$\operatorname{lar}(\mathbf{O})$ and vector $(\mathbf{V})$ characters, they have the form

$$
\Theta_{\mathbf{r}}\left(\tau, z_{i}, 0\right)=\sum_{n_{l}=0,2} \Pi \Theta_{n_{i}, 2}\left(\tau, z_{i}, 0\right),
$$

where for $\mathbf{r}=0$, the sum is further restricted to $\sum n_{i}=0$ $(\bmod 4)$ and for the $\mathbf{r}=\mathbf{V}$ sum is restricted to $\sum n_{i}=2$ $(\bmod 4)$.

The partition function of the theory is a linear combination of the products of the characters discussed above. Each term in the holomorphic part can be labeled by a set of integers $l_{L}=\left[\lambda,\left(l_{i}, q_{i}, s_{i}\right)^{i=1, \ldots, r}\right]$, where $\lambda$ is a weight of $\mathrm{SO}(d)$ at level 1 and $\left(l_{i}, q_{i}, s_{i}\right)$ labels the $r$ th minimal model character. A similar vector $l_{R}$ can be used to label the antiholomorphic part. In order to obtain a consistent solution, one still has to implement $N=1$ superconformal symmetry and possibly space-time supersymmetry. These conditions require extra projections in the partition functions. It is, in general, a hard task to make these projections consistent with modular invariance. However, Gepner has devised a technique called the $\beta$ method that can implement these projections in a modular-invariant fashion.

Under this scheme the type-II partition function can be written as

$\boldsymbol{Z}(\tau, \bar{\tau})=\left(\frac{1}{\operatorname{Im}(\tau)|\eta(\tau)|^{4}}\right)^{d / 2}\left(\frac{1}{2^{r}}\right)\left(\sum_{l, l} N_{l \bar{l}} \boldsymbol{Z}_{V}^{l}(\tau) Z_{\bar{V}}^{\bar{l}}(\tau)\right)$,

where

$$
Z_{V\left(\lambda ; q_{1}, \ldots, q_{r} ; s_{1}, \ldots, s_{r}\right)}^{l\left(l_{1}, l_{2}, \ldots, l_{r}\right)}=\chi_{\lambda}^{\mathrm{SO}(d)} \prod_{i=1}^{r} \chi_{l_{i}, q_{i}}^{k_{i},\left(s_{i}\right)}
$$

and $N_{\overline{l l}}=\prod_{i=1}^{r} N_{l_{i}, \bar{l}_{i}}$ represents a product of affine invariants and $V$ and $\frac{V}{V}$ are $(2 r+1)$-dimensional vectors of the form $\left(\lambda ; q_{1}, \ldots, q_{r} ; s_{1}, \ldots, s_{r}\right)$. The index $\lambda(\bar{\lambda})$ denotes one of the four conjugacy classes $(0, V, s, \bar{s})$ of the $\mathrm{SO}(2 n)$ affine Lie algebra at level 1. The algebra of these classes are $V+s=\bar{s}, V+\bar{s}=s, V+V=0$ for $d=2 n$ and $s+\bar{s}=V, \quad s+s=\bar{s}+\bar{s}=0$ for $d=4 n$ or $s+\bar{s}=0$, $s+\bar{s}=\bar{s}+\bar{s}=V$ for $d=4 n+2$. The $\beta$ method requires that $\bar{V}-V$ belong to a lattice spanned by a set of vectors $\boldsymbol{\beta}_{i}(i=1, \ldots, r)$. The sum over the $\boldsymbol{\beta}_{i}$ lattice implements a generalized Gliozzi-Scherk-Olive ${ }^{13}$ (GSO) projection for the desired symmetry. Modular invariance requires that $\beta_{i}^{2}=$ integer and $\beta_{i} \beta_{j}=$ integer. For $N=1$ superconformal symmetry one has to make sure that the fields in the theory should be either in the Ramond sector of every $N=2$ subtheory (including the space-time part) or in the NS sector of every subtheory simultaneously. This condition can be implemented by $r \beta$ vectors; $\beta_{i}=(\lambda=V ; 0 ; 2$ on $i$ and 0 elsewhere) and at the same time demanding that $s_{i}=1 \bmod 2$ for $\lambda=s, \bar{s}$ and $s_{i}=0$ mode 2 for $\lambda=0, V$ for each $i$ in the product in Eq. (28). For $N=1$ spacetime symmetry, one has to introduce another projection $\beta_{0}=(\lambda=s ; 1, \ldots, 1 ; 1, \ldots, 1)$ and require the $\mathrm{U}(1)$ charge $Q$ associated with the $N=2$ superconformal symmetry to be an odd integer. $Q$ can be calculated as

$$
Q=\sum \lambda_{i}+\sum_{i=1}^{r}\left(-\frac{q_{i}}{k_{i}+2}+\frac{s_{i}}{2}\right) \in(2 Z+1),
$$

where $\sum \lambda_{i}$ is the sum of components of $\mathrm{SO}(d)$ weights. Note that due to the type-II nature of the construction, one automatically obtains an $N=2$ supersymmetry theory.

Having a consistent type-II $N=2$ supersymmetry theory, one can now implement a procedure, we call "heterotic replacement," to convert it into an $N=1 \mathrm{su}$ persymmetric heterotic theory. This is where our earlier discussions on heterotic conformal theory becomes useful. The modular-invariant partition function of our type-II theory can be written as

$$
Z(\tau, \bar{\tau})=\sum_{\lambda, \bar{\lambda}} \chi_{\lambda}^{d}(\tau) \chi_{\bar{\lambda}}^{d}(\bar{\tau}) Z_{\lambda, \bar{\lambda}}(\tau, \bar{\tau}),
$$

where $\lambda, \bar{\lambda}$ labels the level-1 characters of the $G=\mathrm{SO}(d)$ algebra $(0),(V),(s)$, and $(\bar{s})$.

Now, assuming that we have found a heterotic modular-invariant partition function

$$
Z=\sum_{\lambda} \chi_{\lambda}^{d}(\tau) \chi_{\lambda}^{d, e^{*}}(\tau)
$$

for some "effective character"

$$
\chi_{\lambda}^{d, e}(\tau)=q^{h_{\lambda}^{e}-(d+24) / 48} \sum_{n=0}^{\infty} a_{\lambda, n} q^{n}
$$

such that $a_{\lambda, n} \geq 0$ for $\lambda=(0),(V)$ and $a_{\lambda, n} \leq 0$ for $\lambda=(s),(\bar{s})$, where $h_{0}^{e}=1, h_{V}^{e}=0$ and $h_{s, \bar{s}}^{e}=\frac{1}{2}+d / 16$. Equation (29) indicates that the effective characters $\chi_{\bar{\lambda}}^{e}$ transform in the same way as $\chi_{\bar{\lambda}}^{G}$ under modular transformations. Therefore, we can easily convert the partition function of Eq. (29) into a partition function for the 
heterotic string theory:

$$
Z_{\text {het }}=\sum_{\lambda, \bar{\lambda}} \chi_{\lambda}^{d}(\tau) \chi_{\bar{\lambda}}^{d, e^{*}}(\bar{\tau}) Z_{\lambda, \bar{\lambda}}
$$

There are many different ways one can construct the heterotic modular invariants as in Eq. (30). In Sec. III we have exhausted all such invariants which can be constructed using real, free fermionic construction. It is not clear whether or not the complex fermionic construction or bosonic orbifold construction can give rise to new invariants. Note that the condition on the multiplicity coefficient $a_{\lambda, n}$ in $\chi_{\lambda}^{e}(\tau)$ is required by the spin-statistics condition. Since the spin-statistics relation is an automatic consequence of the free fermionic construction, this condition is satisfied by all the solutions of Table I. This is but one of the consequences of string theory. For all the cases considered in Table I, $\chi_{\lambda}^{e}$ turns out to be a linear combination of the characters of an affine Lie algebra $A_{R}$. The model $M_{i}^{D}(i=1,2)$, for $D=4,6,8$ is the one found and used by Gepner.

To work out the consequences of the new construction in the superstring theory, one has to work out the spectrum level by level. It is in principle very difficult to determine whether or not one has a new model if the spectrum of the first few levels happens to be the same as the old one. However, one can in principle identify the gauge symmetry of the theory by the construction of the massless vector fields. It is customarily assumed that if two theories have the same gauge symmetry, and the same tachyonic and massless spectrum, the two theories are probably identical. As an illustrative example, we shall take the $(1)^{9}$ theory with $N_{l l^{\prime}}=\delta_{l l^{\prime}}$ as the internal $N=2$ part of the construction. A complete set of $N=2$, $c=9$ theories that use the tensor products of the minimal theories is available in Ref. 14.

We have worked out the gauge symmetry and the massless vector fields of all the $N=2,(1)^{9}$ models using the heterotic partition function of Table I. It is amazing to find that in each dimension, only two different groups emerge. And even more, all the models in each dimension have the same number of massless fields.

In fact, it is possible to prove ${ }^{15}$ that for each dimension, the $N=1$ space-time supersymmetric models with the same $(2,2)$ internal part have unique partition functions. This statement is independent of the type of $(2,2)$ models we use as internal parts. This result complements a similar situation in $D=10$ dimensions in which for $N=1$ space-time supersymmetric models only two gauge groups, $\mathrm{SO}(32)$ and $\mathrm{E}_{8} \times \mathrm{E}_{8}$, are possible and the two happen to have the same partition function. Somehow, the gauge symmetries, for each $D$, for all the diverse cases in Table I, always enlarge to the two groups of Table II. To understand these enlargements better we shall look at some examples.

Before we start on a specific model, it is useful to list the basic properties of the left-handed $N=1$ superconformal sector. The states are characterized by a Kac-Moody representation, $\lambda=$ singlet $(0)$, vector $(V)$, spinor $(s)$ and antispinor $(\bar{S})$ of $\mathrm{SO}(D-2)$ at level 1 . The other useful properties of the ground state associated with $\lambda$ are weights and charges. They are listed in Table III togeth-
TABLE II. Models of Gepner's construction based on products of $k=1, N=2$ theory and HCFT's in Table I.

\begin{tabular}{lccc}
\hline \hline Dimension & Models & $A_{R}$ & $\begin{array}{c}\text { Gauge } \\
\text { bosons }\end{array}$ \\
\hline$D=4$ & $M_{1}^{4}, M_{3}^{4}$ & $\mathrm{SO}(26) \times \mathrm{U}(1)^{8}$ & 334 \\
& $M_{2}^{4}$ & $\mathrm{E}_{8} \times \mathrm{E}_{6} \times \mathrm{U}(1)^{8}$ & \\
$D=6$ & $M_{1}^{6}, M_{3}^{6}$ & $\mathrm{SO}(28) \times \mathrm{SU}(2) \times \mathrm{U}(1)^{5}$ & 386 \\
& $M_{2}^{6}, M_{4}^{6}$ & $\mathrm{E}_{8} \times \mathrm{E}_{7} \times \mathrm{U}(1)^{5}$ & \\
$D=8$ & $M_{1}^{8}, M_{3}^{8}, M_{5}^{8}$ & $\mathrm{SO}(32) \times \mathrm{SU}(3)$ & 504 \\
& $M_{2}^{8}, M_{4}^{8}$ & $\mathrm{E}_{8} \times \mathrm{E}_{8} \times \mathrm{SU}(3)$ & \\
\hline \hline
\end{tabular}

er with $\Delta_{i}$ and $Q_{i}$, which are the required weights and charges to form massless states.

For the right-handed sector, we shall characterize states by the four "effective" characters $\chi_{\lambda}^{e}$ in Eq. (30) and label them by $\left(0^{e}\right),\left(V^{e}\right),\left(s^{e}\right)$, and $\left(\bar{s}^{e}\right)$. Note that for $M_{1}^{D}$ models $\left(0^{e}\right),\left(V^{e}\right),\left(s^{e}\right),\left(\bar{s}^{e}\right)$ correspond to $(V),(0),-(\bar{s})$, $-(s)$ of $\mathrm{SO}(22+D)$, respectively. Using this notation we shall assign $\left(\lambda^{e}\right)$ the same charge as the corresponding $(\lambda)$ in the left-handed sector. $\beta_{0}$ will be represented by $\left[s^{e},(011)^{9}\right]$ and $\beta_{i}$ by $\left[V^{e},(000) \cdots(002) \cdots(000)\right]$, where $(002)$ appears only in the $i$ th component. With this "effective" notation, the right-handed total charge must be odd, just like the lefthanded sector in order to have space-time supersymmetry and modular invariance. This notation is slightly different from Gepner's, but we believe that this is simpler and less confusion. The $\beta$ vectors actually satisfy the identity

$$
6 \beta_{0}=\sum_{i=1}^{c} \beta_{i}
$$

TABLE III. Conformal weights and charges associated with representations of $\mathrm{SO}(d)$ and weights and charges of the inter-

\begin{tabular}{|c|c|c|c|c|c|}
\hline Dimension & Representation & $\Delta_{L}$ & $Q_{L}$ & $\Delta_{i}$ & $Q_{i}$ \\
\hline \multirow[t]{4}{*}{$D=4$} & 0 & 0 & 0 & $\frac{1}{2}$ & \pm 1 \\
\hline & $V$ & $\frac{1}{2}$ & \pm 1 & 0 & 0 \\
\hline & $s$ & $\frac{1}{8}$ & $\frac{1}{2}$ & $\frac{3}{8}$ & $\frac{1}{2}$ \\
\hline & $\bar{s}$ & $\frac{1}{8}$ & $-\frac{1}{2}$ & $\frac{3}{8}$ & $-\frac{1}{2}$ \\
\hline \multirow[t]{4}{*}{$D=6$} & 0 & 0 & 0 & $\frac{1}{2}$ & \pm 1 \\
\hline & $V$ & $\frac{1}{2}$ & \pm 1 & 0 & 0 \\
\hline & $s$ & $\frac{1}{4}$ & \pm 1 & $\frac{1}{4}$ & 0 \\
\hline & $\bar{s}$ & $\frac{1}{4}$ & 0 & $\frac{1}{4}$ & \pm 1 \\
\hline \multirow[t]{4}{*}{$D=8$} & 0 & 0 & 0 & $\frac{1}{2}$ & \pm 1 \\
\hline & $V$ & $\frac{1}{2}$ & \pm 1 & 0 & 0 \\
\hline & $s$ & $\frac{3}{8}$ & $-\frac{1}{2}$ & $\frac{1}{8}$ & $-\frac{1}{2}$ \\
\hline & $\bar{s}$ & $\frac{3}{8}$ & $\frac{1}{2}$ & $\frac{1}{8}$ & $\frac{1}{2}$ \\
\hline
\end{tabular}
nal sector needed for a massless condition. 
for the $(1)^{c}$ product of minimal models, where $c=15-3 d / 2$. The charge and weights of the righthanded sector are given by $Q_{R}\left(\lambda^{e}\right)=Q_{L}(\lambda)$, $\Delta_{R}\left(0^{e}\right)=\Delta_{L}(V)=\frac{1}{2}$, and for $M_{K}^{D}$ models with $k>1$, $\Delta_{R}\left(\bar{s}^{e}\right)=\Delta_{L}(s)+\frac{1}{2}=\Delta_{R}(s)$, while $\Delta_{R}\left(\bar{s}^{e}\right)=\Delta_{L}(s)+\frac{3}{2}$ $=\Delta_{R}(s)$ for $M_{1}^{D}$ models. The algebra for $\lambda^{e}$ are the same as that for $\lambda$, i.e., $\lambda^{e}+\lambda^{\prime e}=\left(\lambda+\lambda^{\prime}\right)^{e}$. The vacuum energy of the right-handed sector is, of course, -1 instead of $-\frac{1}{2}$ (for the left-handed sector).

The existence of the gravitino in the supersymmetric models can explain part of the symmetry enhancement in Table II. The $l_{L} \times l_{R}$ vector of the gravitino can be represented as $\left[s ;\left(l_{i}, q_{i}, s_{i}\right)^{i=1, \ldots, r}\right]\left[V^{e} ;(0,0,0)^{r}\right]$, where internal weight $\Delta_{i}=(10-D) / 16$ and $Q_{L}$ is odd. Note that $\Delta_{s}=(D-2) / 16$. The existence of this state implies that we will have additional gauge bosons from the states characterized by $\left[V ;(0,0,0)^{r}\right]\left[s^{e} ;\left(l_{i}, q_{i}, s_{i}\right)^{i=1, \ldots, r}\right]$ and $\left[V ;(0,0,0)^{r}\right]\left[-s^{e} ;\left(-l_{i},-q_{i},-s_{i}\right)^{i=1, \ldots, r}\right]$ for models $M_{k}^{D}$ with $k>1$. For $k=1, \Delta_{R}(s)$ turns out to be too large for the states to be massless. For $D=4$, this gravitino argument can already explain all the symmetry enhancement of $M_{2}^{4}$ and $M_{3}^{4}$ models.

For $D=6,-s^{e}=s^{e}$; therefore, the two sets of states from the gravitino argument form a doublet. They carry the charge (of opposite sign) of the $\mathrm{U}(1)_{Q}$, which corresponds to the sum of all the $\mathrm{U}(1)$ charge $Q_{i}$. Luckily, to fit this collection of gauge bosons into a Lie group, we also have two additional gauge bosons represented by $l_{R}=\left[0 ;(1,1,0)^{6}\right]$ with $Q_{i}=\left(\frac{1}{3} \cdots \frac{1}{3}\right), l_{R}=\left[0 ;(1,-1,0)^{6}\right]$, and $Q_{i}=\left(-\frac{1}{3} \cdots-\frac{1}{3}\right)$. Together they enhance the $\mathrm{U}(1)_{Q}$ to semisimple $\mathrm{SU}(2)_{Q}$. This enhancement is responsible for the enhancement of $\mathrm{SO}(12)$ in the $M_{2}^{6}$ model to $\mathrm{E}_{7}$ and $\mathrm{E}_{7}$ in $M_{4}^{6}$ to $\mathrm{E}_{8}$. The symmetry enhancement in $M_{3}^{6}$ is a bit more subtle. The original $\mathrm{SO}(4)$ can be represented by $\mathrm{SU}(2)_{1} \times \mathrm{SU}(2)_{2}$. Two linear combinations of these $\mathrm{SU}(2)_{i}$ and $\mathrm{SU}(2)_{Q}$ combine with $\mathrm{SO}(24)$ to form $\mathrm{SO}(28)$. Since the gravitino argument is independent of the particular $N=2$ product of minimal models we use, we suspect that this $\mathrm{SU}(2)$ enhancement is also independent of the details from the $N=2$ subsectors. We have checked that the same enhancement occurs for $(k=2)^{4}$ construction. However, we do not know how to prove this statement for the general case yet.

For $D=8$, the $N=2$ internal sector is smaller, therefore, we have additional gauge bosons which transform as $\left(0^{e}\right)$ representation of $A_{R}$. For the $M_{1}^{8}$ model, $A_{R}=\mathrm{SO}(30), \quad\left(0^{e}\right)=(V)$. Therefore, these additional gauge bosons absorb a $\mathrm{U}(1)$ from the $N=2$ internal part and combine with the adjoint of $\mathrm{SO}(30)$ to form $\mathrm{SO}(32)$. We also get six additional states whose $Q_{i}$ charges span the roots of an SU(3). The symmetry enhancement of the models $M_{2}^{8}, \ldots, M_{5}^{8}$ is a bit more involved to analyze but has very similar characteristics. The analysis of model $M_{4}^{8}$ is included in Appendix B as an example.

To understand to what extent these symmetry enhancements are independent of details in the $N=2$ subsectors, we worked out the $(2)^{10-D}$ models as well. The result is summarized in Table IV.

A particularly interesting feature appears in $D=8$ theories. In Table I the ranks of $A_{R}$ are $11+D / 2$.
TABLE IV. Models of Gepner's construction using products of $k=2, N=2$ theory.

\begin{tabular}{lccc}
\hline \hline Dimension & Models & $A_{R}$ & $\begin{array}{c}\text { Gauge } \\
\text { bosons }\end{array}$ \\
\hline$D=4$ & $M_{1}^{4}, M_{3}^{4}$ & $\begin{array}{c}\mathrm{SO}(26) \times \mathrm{U}(1)^{6} \\
\boldsymbol{E}_{8} \times \mathrm{E}_{6} \times \mathrm{U}(1)^{5}\end{array}$ & 331 \\
& $M_{2}^{4}$ & & \\
$D=6$ & $M_{1}^{6}, M_{3}^{6}$ & $\mathrm{SO}(28) \times \mathrm{SU}(2) \times \mathrm{U}(1)^{3}$ & 384 \\
& $M_{2}^{6}, M_{4}^{6}$ & $\mathrm{E}_{8} \times \mathrm{E}_{8} \times \mathrm{U}(1)^{3}$ & \\
$D=8$ & $M_{1}^{8}, M_{3}^{8}, M_{5}^{8}$ & $\mathrm{SO}^{8}(32) \times \mathrm{SU}(2) \times \mathrm{SU}(2)$ & 502 \\
& $M_{2}^{8}, M_{4}^{8}$ & $\mathrm{E}_{8} \times \mathrm{E}_{8} \times \mathrm{SU}(2) \times \mathrm{SU}(2)$ & \\
\hline \hline
\end{tabular}

Therefore naively one expects the rank of the superstring theory to be $11+D / 2+(10-D)=21-D / 2$ for models with $(2)^{10-D}$ construction. This is the case for $D=4$ and 6. However, for $D=8$, the rank is enhanced by one unit compared with a naive value. This is because there is one additional gauge boson characterized by $l_{R}=\left[V^{e} ;(2,0,0)^{2}\right]$ which does not carry any charge of $Q_{i}$ or $A_{R}$. The possibility of rank enhancement was hinted by Lutken and Ross but it was never worked out explicitly.

Observation of Tables II and IV indicates that our more general heterotic replacements do not produce any new gauge groups that are different from those that can be produced by Gepner's two heterotic replacements. This is a surprising result. However, this does not mean that our efforts are totally useless. It is easy to show that for nonsupersymmetric models, our procedure indeed produces new gauge symmetries. An example is presented in Appendix $\mathrm{C}$ and a list, presumably exhaustive, of such models is given in Table $\mathrm{V}$. In Table $\mathrm{V}$, we have limited ourselves only to the models with $k=1$ minimal $N=2$ theories. Had we not, the list would have been much larger.

Recently, it was proposed ${ }^{16}$ that additional twists may be added to $(2,2)$ models to produce new $(0,2)$ models. These new constructions may be combined with our heterotic replacements to generate new models that cannot be produced otherwise.

\section{CONCLUSION}

We have shown that the construction of heterotic conformal field theory is indeed interesting, useful, and nontrivial. As a first attempt, a free fermionic construction technique is employed to generate many new theories of this type. The technique is then applied to Gepner's construction. Unfortunately, for the $(2,2)$ models with space-time supersymmetry, our new construction simply reproduces the gauge groups that can be produced by using the well-known heterotic invariants of $\mathrm{SO}(d+24)$ and $\mathrm{E}_{8} \times \mathrm{SO}(d+8)$. However, for the nonsupersymmetric case, our procedure generates many new solutions, and thus signals that this new construction may prove to be useful in other applications as well. 
TABLE V. Models in nonsupersymmetric case.

\begin{tabular}{|c|c|c|c|c|}
\hline Dimension & Model & Tachyon & $A_{R}$ & $\begin{array}{l}\text { Gauge } \\
\text { boson }\end{array}$ \\
\hline \multirow[t]{3}{*}{$D=4$} & $M_{1}^{4}$ & 26 & $\mathrm{SO}(26) \times \mathrm{U}(1)^{9}$ & 334 \\
\hline & $M_{2}^{4}$ & 10 & $\mathrm{E}_{8} \times \mathrm{SO}(10) \times \mathrm{U}(1)^{9}$ & 302 \\
\hline & $M_{3}^{4}$ & 2 & $\mathrm{SO}(2) \times \mathrm{SO}(24) \times \mathrm{U}(1)^{9}$ & 286 \\
\hline \multirow[t]{4}{*}{$D=6$} & $M_{1}^{6}$ & 28 & $\mathrm{SO}(28) \times \mathrm{U}(1)^{6}$ & 384 \\
\hline & $M_{2}^{6}$ & 12 & $\mathrm{E}_{8} \times \mathrm{SO}(12) \times \mathrm{U}(1)^{6}$ & 320 \\
\hline & $M_{3}^{6}$ & 4 & $\mathrm{SO}(4) \times \mathrm{SO}(24) \times \mathrm{U}(1)^{6}$ & 288 \\
\hline & $M_{4}^{6}$ & 0 & $\mathrm{E}_{7} \times \mathrm{E}_{7} \times \mathrm{U}(1)^{6}$ & 272 \\
\hline \multirow[t]{5}{*}{$D=8$} & $M_{1}^{8}$ & 30 & $\mathrm{SO}(30) \times \mathrm{U}(1)^{3}$ & 438 \\
\hline & $M_{2}^{8}$ & 14 & $\mathrm{E}_{8} \times \mathrm{SO}(14) \times \mathrm{U}(1)^{3}$ & 342 \\
\hline & $M_{3}^{8}$ & 6 & $\mathrm{SO}(6) \times \mathrm{SO}(24) \times \mathrm{U}(1)^{3}$ & 294 \\
\hline & $M_{4}^{8}$ & 2 & $\mathrm{E}_{7} \times \mathrm{E}_{7} \times \mathbf{U}(1) \times \mathbf{U}(1)^{3}$ & 270 \\
\hline & $M_{5}^{8}$ & 0 & $\mathrm{SU}(16) \times \mathrm{U}(1)^{3}$ & 258 \\
\hline
\end{tabular}

\section{APPENDIX A: FREE FERMIONIC CONSTRUCTION}

In this Appendix we provide a short review and an explicit example of the free fermionic construction. In particular, the example with the gauge group $\mathrm{SO}(6) \times \mathrm{SO}(24)$ in model $M_{3}^{8}$ (Table I) will be worked out in detail.

To describe the solutions of the modular-invariance condition, we have to introduce some notation. As described in Sec. III, the set of boundary conditions can be described by a collection of subsets of fermions. The consistency requires that this collection form a group under the "symmetric difference" operation:

$$
\alpha \beta=\alpha \cup \beta-\alpha \cap \beta .
$$

Under this operation, the collection can be described by some of its elements which serve as the basis elements. Let us say there are $N$ of them, which we denote by $b_{i}$, $i=0,1, \ldots, N$. All the other elements in the collection can be generated from $b_{i}$ by symmetric difference operation. Define a number operator $n(\alpha)=n_{L}(\alpha)-n_{R}(\alpha)$, where $n_{L}(\alpha)$ and $n_{R}(\alpha)$ are the numbers of left- and right-moving fermions in $\alpha$. Modular invariance requires that (1) $\Delta c=c_{L}-c_{R}=0(\bmod 12)$; (2) the set that contains all the fermions $F$ has to be $\Xi$ and we can use it as one of the bases, denoted as $b_{0} ;(3) n\left(b_{i}\right)=0(\bmod 8) ;(4)$ $n\left(b_{i} \cap b_{j}\right)=0(\bmod 4) ;$ and $(5) n\left(b_{i} \cap b_{j} \cap b_{k}\right)=0(\bmod 2)$. For heterotic string models, $\Delta c=12$. We shall restrict ourselves to this case from now on because this is the one applied in Sec. IV. With this collection of sets we can write the modular-invariant partition function as

$$
Z=\sum_{\alpha, \beta \in \equiv} C_{(\alpha \mid \beta)} Z_{C}[\alpha \mid \beta],
$$

where $C_{(\alpha \mid \beta)}$ are coefficients with values $1,-1$ to be determined later. The $Z_{C}$ functions are defined by

$$
Z_{C}[\alpha \mid \beta]=\prod_{f=1}^{n_{L}+n_{R}} Z_{f}\left[a_{f} \mid b_{f}\right],
$$

where $a_{f}$ is 1 if $f$ belongs to $\alpha$ and zero otherwise. For each fermion $Z_{f}$ can be expressed in terms of Jacobi $\theta$ functions and Dedekind $\eta$ functions:

$$
\begin{aligned}
& Z_{f}[0 \mid 0]=\left(\frac{\theta_{3}(\tau)}{\eta(\tau)}\right)^{1 / 2}, \\
& Z_{f}[0 \mid 1]=\left(\frac{\theta_{4}(\tau)}{\eta(\tau)}\right)^{1 / 2}, \\
& Z_{f}[1 \mid 0]=\left(\frac{\theta_{2}(\tau)}{\eta(\tau)}\right)^{1 / 2}, \\
& Z_{f}[1 \mid 1]=\left(\frac{\theta_{1}(\tau)}{\eta(\tau)}\right)^{1 / 2}=0 .
\end{aligned}
$$

To write down the modular invariant solution, we introduce the following notation from ABK. For $X \in \Xi$

$$
\epsilon_{X}=\exp \left(\frac{i \pi}{8} n(X)\right)
$$

and

$$
\delta_{X}= \begin{cases}-1 & \text { if } S \subset X, \\ +1 & \text { if } S \cap X=\emptyset,\end{cases}
$$

where $S=\left\{\chi_{L}^{i}, i=1, \ldots, r\right\}$ and a parity operator that counts the fermions in $X(\bmod 2)$,

$$
(-)^{X} f= \begin{cases}-f(-)^{X} & \text { if } f \in X, \\ f(-)^{X} & \text { if } f \notin X .\end{cases}
$$

Given conditions (1)-(5) above, one can choose each of the coefficients $C_{(F \mid F)}$ and $C_{\left(b_{\imath} \mid b_{j}\right)}$ for $i>j$ to be \pm 1 . All other $C_{(\alpha \mid \beta)} \in \Xi$ are determined by using the properties

$$
\begin{aligned}
& C_{(\alpha \mid 0)}=\delta_{\alpha}, \\
& C_{(\alpha \mid \beta)}=\epsilon_{\alpha \cap \beta}^{2} C_{(\beta \mid \alpha)}, \\
& C_{(\alpha \mid \alpha)}=-\epsilon_{\alpha} C_{(\alpha \mid F)}, \\
& C_{(\alpha \mid \beta)} C_{(\alpha \mid \gamma)}=\delta_{\alpha} C_{(\alpha \mid \beta \gamma)} .
\end{aligned}
$$


Each of the $2^{N(N+1) / 2+1}$ choices of $C_{(F \mid F)}$ and $C_{\left(b_{i} \mid b_{j}\right)}$ define a modular invariant partition function

$$
Z=\frac{1}{2^{N+1}} \sum_{\alpha, \beta \in \Xi} C_{(\alpha \mid \beta)} Z_{C}[\alpha \mid \beta] .
$$

Now we shall use this machinery to identify all the massless gauge-boson states and the representations in the model $M_{3}^{8}$. As mentioned earlier, the gauge group in this case is $\mathrm{SO}(24) \times \mathrm{SO}(6)$.

This gauge group results when $F=\left(\chi_{R}^{1} \cdots \chi_{R}^{30}\right)$, $b_{1}=\left(\chi_{R}^{1} \cdots \chi_{R}^{16}\right)$, and $b_{2}=\left(\chi_{R}^{1} \cdots \chi_{R}^{8}, \chi_{R}^{17} \cdots \chi_{R}^{24}\right)$ are chosen as bases.

We can construct eight different sectors out of these three bases by means of symmetric difference operation discussed earlier in this Appendix. They are of the form $b_{i}, b_{i} b_{j}$, and $b_{i} b_{j} b_{k}(i, j, k=0,1,2)$. However, because of the mass-shell condition

$$
\begin{aligned}
M^{2} & =-\frac{1}{2}+\frac{n_{L}(\alpha)}{16}+\sum(\text { frequencies }) \\
& =-1+\frac{n_{R}(\alpha)}{15}+\sum(\text { frequencies })
\end{aligned}
$$

massless bosonic states can appear only in $\phi, b_{1}, b_{2}$, and $b_{1} b_{2}$ sectors. Other sectors contribute only to massive states. There is another set of constraints that come from the requirement of modular invariance. Each state in sector $\alpha$ should survive the following GSO projection by every basis vector $b_{i}$ :

$$
C_{\left(\alpha \mid b_{i}\right)} \delta_{\alpha}(-1)^{b_{t}}=1 \text {. }
$$

The effect of making a $\beta$ projection $(-1)^{\beta}$ in the sector $\alpha$ depends on whether or not $\alpha \cap \beta$ is empty. If it is empty, then the projection is +1 for those states that are built by an even number of oscillators on the ground state $|\alpha\rangle$ and $(-1)$ on the states that are built by odd number of oscillators. Hence the $\beta$ projection works simply as an operator $(-1)^{\mathcal{F}}$, where $\mathcal{F}$ is the fermion number operator. However if $\alpha \cap \beta$ is not empty, then one defines a generalized chirality operator

$$
\Gamma_{\alpha \cap \beta}=\prod_{f \in \alpha \cap \beta} f_{0}
$$

where $f_{0}$ is the zero mode of the fermions in $\alpha \cap \beta$, and the action of $(-1)^{\beta}$ is given by the product of the $(-1)^{\mathcal{F}}$ and chirality operator on the state.

Now we will analyze all sectors to identify the gauge bosons and the representations they come in.

$\Phi$ sector. In this sector all the fermions are antiperiodic. Let us denote the ground state in this sector by $|\Phi\rangle$. Vector bosons are then given by $\psi^{\mu} \chi_{R}^{i} \chi_{R}^{j}|\Phi\rangle$. The projection by sector $b_{1}$ demands that $\chi_{R}^{i}$ and $\chi_{R}^{i}$ be outside the set $b_{1}$ or inside for this state to survive the GSO projection. Similar constraints are forced by sector $b_{2}$ projection. Thus $b_{1}$ and $b_{2}$ projections together make sure only those states survive that are created by choosing $\chi_{R}^{i}$ to be in one of the following sets: $\left(\chi_{R}^{1} \cdots \chi_{R}^{8}\right)$, $\left(\chi_{R}^{9} \cdots \chi_{R}^{16}\right),\left(\chi_{R}^{17} \cdots \chi_{R}^{24}\right),\left(\chi_{R}^{25} \cdots \chi_{R}^{30}\right)$. Thus gauge bosons in this sector naturally arise in the representation
$(28,1,1,1),(1,28,1,1$,$) , and (1,1,28,1)$ of $(\mathrm{SO}(8))^{3} \times \mathrm{SO}(6)$.

$b_{1}, b_{2}, b_{1} b_{2}$ sectors. First the $b_{1}$ sector; this sector has 16 periodic right-handed fermions and consequently vacuum $\left|b_{1}\right\rangle$ itself saturates the massless conditions. The gauge bosons are given by $\psi^{\mu}\left|b_{1}\right\rangle$. This ground state is an $\mathrm{SO}(16)$ spinor and each state can be represented by a vector of the type

$$
\left|s_{1}\right\rangle \equiv\left( \pm \frac{1}{2}, \pm \frac{1}{2}, \pm \frac{1}{2}, \pm \frac{1}{2}, \pm \frac{1}{2}, \pm \frac{1}{2}, \pm \frac{1}{2}, \pm \frac{1}{2}\right) \text {. }
$$

The chirality of the state is defined by $(-1)^{N}$, where $N$ is the number of negative components in this vector. The $b_{1}$ projection implies that only one overall chirality survives. Without loss of generality we can take it to be positive. Hence only an even number of negative components are allowed. Now the $b_{2}$ projection constrains the product of the last four components to have a fixed sign. Thus there are a total of $\frac{1}{2} \times \frac{1}{2} \times 2^{8}=2^{6}$ states in this sector and they transform as $(8,8,1,1)$ under $(\mathrm{SO}(8))^{3} \times \mathrm{SO}(6)$. For $b_{2}$ and $b_{1} b_{2}$ sectors, in an exactly analogous way, we find that there are 64 states in each of them and they transform as $(8,1,8,1)$ and $(1,8,8,1)$, respectively. Thus these states from all four sectors combine to form the adjoint representation of $\mathrm{SO}(24) \times \mathrm{SO}(6)$.

\section{APPENDIX B: AN EXAMPLE OF GEPNER'S CONSTRUCTION}

In this Appendix we are going to describe an example, the $M_{4}^{8}$ model of Table II, to demonstrate the $N=2$ model construction and how enlargement of the gauge group results. The example we use here is typical of all other models in text.

We will work in eight space-time dimensions and choose four bases $F, b_{1}, b_{2}, b_{3}$. The right-handed characters are characters of the Lie algebra $G=\mathrm{E}_{7} * \mathrm{E}_{7} * \mathrm{U}(1)$. In this case the contribution to $c$ coming from the $N=2$ part is 3 . There are three different ways this can be achieved. In this model we achieve this result by taking three copies of $c=1$ theories.

We will be only concerned with states which represent massless vector bosons. The left-moving part of these states should be $\left[V ;(0,0,0)^{3}\right]$. Here the first component $V$ indicates that it is a vector boson. Since the weight, $\frac{1}{2}$, of the vector state of $\mathrm{SO}(2 n)$ already saturates the massless condition, the $N=2$ part is constrained to have weight zero. This fixes their quantum numbers to be $(0,0,0)^{3}$. The right-moving part then will start with exactly same $N=2$ part except the first component will be replaced by $V^{e}$ due to heterotic replacement. The massless condition requires the right-moving sector to have weight $\Delta$ equal to 1 . The quantum numbers of the allowed right-moving states will live on a lattice that is generated by the basis vectors $\beta_{0}$ and $\beta_{i}$ starting from the vector $\left[V^{e},(0,0,0)^{3}\right]$. The $\beta$ vectors $\beta_{0}, \beta_{i}$ are given by $\left[s^{e} ;(0,1,1)^{3}\right]$ and $\beta_{i}=\left[0^{e} ;(0,0,2)_{i}\right]$, respectively. The space-time supersymmetry also requires that the total $\mathrm{U}(1)$ charge of each bonafide state to be odd. When all thees conditions are imposed, the entire lattice breaks down into following classes.

(1) [Adjoint of $E_{7} \times E_{7} \times U(1)^{4} ;(0,0,0)^{3}$ ]. This state is obtained by applying $\mathrm{E}_{7} * \mathrm{E}_{7} * \mathrm{U}(1)$ currents on the state 
$\left[V^{e},(0,0,0)^{3}\right]$. The multiplicity is $133+133+1=267$. Three similar states can be obtained by applying the $\mathrm{U}(1)$ currents of $N=2$ theory on $\left[V^{e},(0,0,0)^{3}\right]$.

(2) $\left[s^{e} ;(0,1,1)^{3}\right]$ and $\left[\bar{s}^{e} ;(0,5,-1)^{3}\right]$. The number of massless states in these sectors can be derived from the general character formula in Eqs. (8)-(10) by expanding these characters in powers of $q$ and finding the multiplicities of the term that correspond to the massless states. In this case it is 112 .

(3) $\left[0^{e} ;(0,2,2)^{3}\right]$ and $\left[0^{e} ;(0,4,2)^{3}\right]$. By the same procedure as in class (2), we found the multiplicity of these states to be 2 .

(4) $\left[V^{e} ;(0,2,0)(0,2,2)^{2}\right]$ and $\left[V^{e} ;(0,4,0)(0,4,2)^{2}\right]$. These states contribute six gauge bosons because there are three different ways of permuting the $N=2$ part. Note that $V^{e}$ is actually a singlet of $G$.

When all contributions are added up, we get a total of 504 massless vector bosonic states. A more careful analysis of their weight vectors shows that they fit nicely into the Lie algebra $\mathrm{E}_{8} * \mathrm{E}_{8} * \mathrm{SU}(3)$.

\section{APPENDIX C: A NONSUPERSYMMETRIC EXAMPLE}

In this Appendix we work out a nonsupersymmetric example. We will limit the discussion to models using only $k=1$ minimal $N=2$ theory. In Gepner's construction of supersymmetric theories the difference $\bar{V}-V$ between the right-moving quantum numbers $\bar{V}$ and leftmoving quantum numbers $V$ is generated by a lattice spanned by $\beta_{0}$ and $\beta_{i}$. The space-time supersymmetry is guaranteed by the inclusion of $b_{0}$ and requiring the total $\mathrm{U}(1)$ charge to be odd. The two conditions are related by a modular transformation. If we relax the condition of supersymmetry, we should drop both of these conditions.

We are only interested in massless vector bosons just as in Appendix B. In the four-dimensional case, the lefthanded sector will have the quantum numbers $\left[V ;(0,0,0)^{9}\right]$. For the right-handed part we start with the vector $\left[V^{e} ;(0,0,0)^{9}\right]$ and generate all the other states by adding $\beta_{i}$. The state $\left[V^{e} ;(0,0,0)\right]$ has $\Delta=0$; hence, the massless states can be obtained in this sector by operation with the $\mathrm{U}(1)$ current of each of the nine $N=2$ subsectors or the currents of the gauge group $G$.

Take the model $M_{1}^{4}$ of Table I with $G=\mathrm{SO}(26)$, for example. No massless state is obtained by applying $\beta_{i}$ on $\left[V^{e} ;(0,0,0)^{9}\right]$. Thus we see that there is no symmetry enlargement in this case and the gauge group after including internal $(N=2)$ degrees of freedom is $\mathrm{SO}(26) * \mathrm{U}(1)^{9}$. The non-Abelian part of the gauge group remains the same as the one obtained from the free fermionic construction. This pattern is quite general and is repeated for all other models of Table $I$. This is distinctively different from the supersymmetric case where symmetry enlargement was a common feature. Therefore for nonsupersymmetric cases our new heterotic maps produce models that have different gauge groups compared with Gepner's heterotic replacements. They are listed in Table V.
${ }^{1}$ For a review, see M. Green, J. Schwarz, and E. Witten, Superstring Theory (Cambridge University Press, Cambridge, England, 1987), Vols. 1 and 2.

${ }^{2}$ P. Candelas, G. Horowitz, A. Strominger, and E. Witten, Nucl. Phys. B258, 46 (1985).

${ }^{3}$ L. Dixon et al., Nucl. Phys. B261, 678 (1985); B274, 285 (1986); L. Dixon and J. Harvey, ibid. B274, 193 (1986); L. Alvarez-Gaume et al., Phys. Lett. B 171, 155 (1986).

${ }^{4}$ W. Lerche, A. Schellekens, and N. Warner, Phys. Rep. 177, 1 (1989), and references therein.

${ }^{5}$ H. Kawai, D. Lewellen, and S. Tye, Phys. Rev. Lett. 57, 1832 (1987); Phys. Rev. D 34, 3794 (1986); Nucl. Phys. B288, 1 (1987); Int. J. Mod. Phys. A 3, 279 (1988); I. Antoniadis, C. Bachas, and C. Kounnas, Nucl. Phys. B289, 87 (1987); S. Ferrara and C.Kounnas, ibid. B328, 406 (1989); C. S. Lam, Int. J. Mod. Phys. A 3, 913 (1988); N. Seiberg and E. Witten, Nucl. Phys. B276, 272 (1986).

${ }^{6}$ D. Gepner, Nucl. Phys. B296, 757 (1988); Phys. Lett. B 199, 380 (1987).

${ }^{7}$ D. Kastor, E. Martinec, and Z. Qiu, Phys. Lett. B 200, 434 (1988).

${ }^{8}$ G. Moore and N. Seiberg, Report No. IASSNS-HEP-88/39 (unpublished); L. Alvarez-Gaume, C. Gomez, and G. Sierra, Report No. CERN-TH. 5129/88 (unpublished).

${ }^{9}$ D. Gross. J. Harvey, E. Martinec, and R. Rohm, Phys. Rev. Lett. 54, 502 (1985); Nucl. Phys. B256, 253 (1985); B267, 75 (1986); K. S. Narain, Phys. Lett. 169B, 41 (1986).

${ }^{10}$ T. Banks, L. Dixon, D. Friedan, and E. Martinec, Nucl. Phys. B299, 601 (1988); T. Banks and L. Dixon, ibid. B307, 93 (1988).

${ }^{11}$ F. Ravanini and S. K. Yang, Phys. Lett. B 195, 202 (1987); Z. Qiu, ibid. 198, 497 (1987); Y. Matuo, Prog. Theor. Phys. 77, 93 (1987); V. K. Dobrev, Phys. Lett. B 186, 43 (1987).

${ }^{12}$ V. Kac and H. Peterson, Adv. Math. 53, 125 (1984).

${ }^{13}$ F. Gliozzi, J. Scherk, and D. Olive, Nucl. Phys. B122, 253 (1977).

${ }^{14}$ C. Lutken and G. Ross, Phys. Lett. B 213, 152 (1988); M. Lynker and R. Schimmerigk, ibid. 215, 681 (1988).

${ }^{15}$ D. Chang, A. Kumar, and B. Rostand, Phys. Lett. B 243, 80 (1990).

${ }^{16}$ A. Font, L. E. Ibanez, M. Mondragon, F. Quevedo, and G. G. Ross, Phys. Lett. B 227, 34 (1989); A. Font, L. E. Ibanez, F. Quevedo, and A. Sierra, Nucl. Phys. B337, 119 (1990). 